\title{
Risk factors of postoperative nausea and vomiting after total hip arthroplasty or total knee arthroplasty: a retrospective study
}

\author{
Yingjie Wang", Qi Yang", Jin Lin, Wenwei Qian, Jin Jin, Peng Gao, Baozhong Zhang, Bin Feng, \\ Xisheng Weng
}

Department of Orthopedic Surgery, Peking Union Medical College Hospital, Chinese Academy of Medical Sciences \& Peking Union Medical College, Beijing, China

Contributions: (I) Conception and design: Y Wang, X Weng; (II) Administrative support: Q Yang; (III) Provision of study materials or patients: J Lin, W Qian; (IV) Collection and assembly of data: J Jin, P Gao, B Zhang; (V) Data analysis and interpretation: Y Wang, B Feng; (VI) Manuscript writing: All authors; (VII) Final approval of manuscript: All authors.

\#The authors contributed equally to this work.

Correspondence to: Bin Feng; Xisheng Weng. Department of Orthopedic Surgery, Peking Union Medical College Hospital, Chinese Academy of Medical Sciences \& Peking Union Medical College, Beijing, China. Email: pumcfeng@163.com; xshweng@medmail.com.cn.

\begin{abstract}
Background: Postoperative nausea and vomiting (PONV) is a common complication after total hip/knee arthroplasty (THA/TKA) that affects patient satisfaction and postoperative recovery. It has been reported that patients undergoing THA/TKA experience PONV at a frequency of $20-83 \%$. This study investigates the occurrence of PONV in patients and analyzes the risk factors.

Methods: Patients undergoing primary THA/TKA under general anesthesia from October 1, 2017, to May 1, 2018, were included. Data on patient-related factors were collected before THA/TKA. Anesthesiaand surgery-related factors were recorded postoperatively. Risk factors were analyzed using binary logistic regression.

Results: A stronger association of motion sickness and PONV was found at six hours after bilateral THA/ TKA [nausea: odds ratio $(O R)=14.648,3.939-54.470$; vomiting: $O R=8.405,2.482-28.466]$. At 6-24 hours after bilateral THA/TKA, patients who had a history of migraines tended to experience nausea $(\mathrm{OR}=12.589,1.978-$ 80.105). Patients with lower body mass index (BMI) were more likely to experience PONV at 24-72 hours (nausea: $\mathrm{OR}=0.767,0.616-0.954$; vomiting: $\mathrm{OR}=0.666,0.450-0.983$ ) after bilateral THA/TKA.

Conclusions: The incidence of PONV after primary bilateral THA/TKA was higher than that after unilateral THA/TKA. The risk factors vary at different time points after surgery, and a history of motion sickness is the most critical factor affecting PONV.
\end{abstract}

Keywords: Postoperative nausea and vomiting (PONV); total hip arthroplasty (THA); total knee arthroplasty (TKA); risk factors

Submitted Jul 13, 2020. Accepted for publication Aug 25, 2020.

doi: 10.21037/atm-20-5486

View this article at: http://dx.doi.org/10.21037/atm-20-5486

\section{Introduction}

Postoperative nausea and vomiting (PONV) remain one of the most common anesthesia-related complications (1). Total hip arthroplasty (THA) and total knee arthroplasty
(TKA) are effective methods for the treatment of end-stage hip and knee joint diseases, respectively, that can effectively relieve pain, improve joint function, and enhance the patients' quality of life (2). It has been reported that patients undergoing THA/TKA experience PONV at a frequency of 
$20-83 \%(3,4)$. In most patients, mild PONV causes serious discomfort; however, in severe cases, PONV may increase the risk of pulmonary thromboembolism, dehydration, and water and electrolyte imbalance, and might also interfere with rehabilitation, thus increasing the patients' burden, hospital stay, and medical costs, leading to poor clinical outcome and patient dissatisfaction after THA/TKA (5-7). Therefore, it is crucial to investigate the risk factors affecting PONV after THA/TKA. Multiple interventions can be used in the perioperative period to reduce the incidence of PONV and improve patient satisfaction.

It is currently believed that there are multiple factors from three aspects affecting PONV from published studies (8). However, there is still insufficient one study systematically analyzing the effects of all the multiple factors from three aspects of PONV. First, the first aspect is patient-related factors including sex, age, obesity, history of smoking, and history of motion sickness. Then, the second aspect is anesthesia-related factors, including the dosage of anesthetic drug and anesthesia time. Last, the third aspect is surgical-related factors, including operation time and surgical planning, including unilateral and spontaneous bilateral THA/TKA. In recent years, many studies have focused on the risk factors of PONV (9-11). However, most studies focused on a single factor. For example, Ukai et al. found that the incidence of PONV was associated with female sex, body mass index (BMI), and use of opioids through an epidural catheter (12). To our knowledge, few studies have evaluated the association between many factors and PONV after THA/TKA.

Previous studies demonstrated that bilateral TKA resulted in more complications than unilateral TKA and that patients who underwent unilateral TKA had a markedly lower average pain than those who underwent simultaneous bilateral TKA replacements on day one after surgery $(13,14)$. Further, the operation time and anesthesia time were different between patients who underwent unilateral surgery and those who underwent bilateral surgery. Therefore, in the present study, we divided all patients into two groups according to unilateral or bilateral TKA/THA and analyzed the risk factors of PONV at different time points (0-6, 6-24, and 24-72 hours) postoperatively, to provide evidence that would help clinicians reduce the incidence of postoperative PONV.

We present the following article in accordance with the STROBE reporting checklist (available at http://dx.doi. org/10.21037/atm-20-5486).

\section{Methods}

\section{Patients}

Consecutive patients who underwent primary THA or TKA from October 1, 2017, to May 1, 2018, at our medical center retrospectively were investigated. The preoperative diagnoses included developmental dysplasia of the hip, aseptic femoral head necrosis, femoral neck fracture, osteoarthritis, rheumatoid arthritis, and others. Patients were undergoing revision THA or TKA, undergoing primary THA or TKA under neuraxial anesthesia, and using antiemetic drugs before surgery is excluded. Patients with clarified gastritis or gastric ulcer were also excluded. The authors did not have access to information that could identify individual participants during or after data collection. The medical data in this study has been approved by the Institutional Review Board (IRB) of Peking Union Medical College Hospital. All procedures performed in this study involving human participants were in accordance with the Declaration of Helsinki (as revised in 2013). Because of the retrospective nature of the research, the requirement for informed consent was waived.

\section{Operations}

All patients were placed in the lateral or supine position on a standard orthopedic table. Then, the THA and TKA were performed through a posterolateral approach and medial parapatellar approach, respectively. After that, the patients received general intravenous anesthesia during the surgery. The preoperative anesthetic drug includes propofol, sevoflurane, fentanyl, remifentanil, and rocuronium. Many measures were taken to minimize the blood loss, including using a tourniquet and postoperative pressure bandaging for TKA and using tranexamic acid and electric coagulation hemostasis, even peratriction during the surgery of both TKA and THA. The patient started using the erythropoietin and low molecular weight heparin sodium from the first day after surgery until she was discharged, and iron sucrose from the first day to the third day postoperatively.

The postoperative analgesic drug in a venous analgesic pump includes morphine, remifentanil, or oxycodone, and saline. The analgesia pump had a background speed of $0-4 \mathrm{~mL} /$ hour. Last, the total volume of the analgesia pump is $250 \mathrm{~mL}$. The amount of oxycodone and remifentanil was converted to the dosage of morphine, according to the 
Handbook of Clinical Anesthesia Procedures of the Massachusetts General Hospital. Cementless femoral stem (CORAIL; Depuy, France), acetabular shell sector (PINNACLE, Depuy), ceramic insert (BIOLOX, Depuy), and ceramic femoral head (BIOLOX, Depuy) components were used in patients who underwent THA to compare the dosage of anesthetic agents in the pump quantitively. Nonporous femoral component (Smith \& Nephew, UK), high flexion articular insert (Smith \& Nephew), and nonporous tibial baseplate (Smith \& Nephew) were used in patients who underwent TKA.

\section{Preoperative examination}

BMI (an index for overall obesity) was calculated as body weight divided by height (in square meters). All patients were needed to have an empty stomach 6-8 hours before surgery. The preoperative hemoglobin $(\mathrm{Hb})$ level was evaluated with Bayer ADVIA 2120 (Germany). Information on the history of smoking, migraine, motion sickness, and preoperative oral glucocorticoid use was recorded.

\section{Study definition and data collection after the operation}

Obesity was defined as a BMI of $\geq 30 \mathrm{~kg} / \mathrm{m}^{2}$. The postoperative $\mathrm{Hb}$ level was evaluated with Bayer ADVIA 2120 (Germany) at 24 and 72 hours after the operation. Then, the patients scored their pain level on a visual analog scale (VAS) at 6 and 24 hours after the operation. Also, nonvomiting was set to 0 , while vomiting was set to 1 . The vomiting score after 72 hours was calculated as follows: $1 \times$ vomiting frequency ( 6 hours) $+1 \times$ vomiting frequency ( $6-24$ hours $)+$ $1 \times$ vomiting frequency (24-72 hours). The data of patients were analyzed at $0-6,6-24$, and $24-72$ hours, respectively, to analyze the risk factors of $\mathrm{PONV}$ at different time points after THA/TKA.

\section{Statistical analysis}

Normally distributed continuous variables (BMI, preoperative $\mathrm{Hb}, \mathrm{Hb}$ at 24 hours after surgery, and $\mathrm{Hb}$ at 72 hours after surgery) are presented as means \pm standard deviations and are analyzed using an independent $t$-test. Variables with a skewed distribution (age, anesthesia time, VAS, vomiting score, and analgesic morphine consumption) are presented as median (interquartile range) and are compared using the Mann-Whitney U-test. Categorical data (sex, motion sickness, migraine, history of oral glucocorticoid use, analgesia pump use) are presented as percentages and are compared using the $\chi^{2}$ test and fisher's exact test. Binary logistic regression analyses were performed to calculate the odds ratios (ORs) of risk factors according to either nausea or vomiting, with adjustments for related confounding factors. All $\mathrm{P}$ values are two-sided, and $\mathrm{P}<0.05$ is considered statistically significant. All statistical analyses were performed using SPSS 20.0 software (SPSS Inc., Chicago, IL, USA).

\section{Results}

\section{Overview of patients}

The study included 673 affected joints in 496 patients (176 joints in men and 497 joints in women). Of all patients, 314 underwent primary TKA (69 joints in men and 380 joints in women), and 182 patients underwent primary THA (107 joints in men and 117 joints in women). Three hundred nineteen patients underwent unilateral THA/TKA, and 177 patients underwent spontaneous bilateral THA/TKA.

After unilateral THA/TKA, the nausea frequency peaked at $14.73 \%$ at 6 hours, and the vomiting frequency peaked at $7.52 \%$ at $6-24$ hours $(\mathrm{P}<0.05)$. After bilateral THA/TKA, the frequency of nausea and vomiting peaked at $22.60 \%$ and $14.69 \%$, respectively, at $0-6$ hours. The results are shown in Table 1 and Figure 1.

\section{General characteristics of patients at different time points after THA/TKA}

Four hundred ninety-six patients were grouped according to whether unilateral or bilateral THA/TKA was performed and whether PONV occurred at 6, 6-24, and 24-72 hours after surgery. The basic data of patients within 0-6, 6-24, 24-72 hours after THA/TKA including the information about patients' age, sex, BMI, Obesity, Smoking, Anesthesia time, Preoperative $\mathrm{Hb}, \mathrm{Hb}$ after 24 hours, Hb after 72 hours, motion sickness, migraine, preoperative oral glucocorticoid, VAS at 6 hours, VAS after 24 hours, analgesia pump, morphine consumption 0-6 hours, morphine consumption within 24 hours, and morphine consumption within 72 hours were analyzed among which the remarkable data or differences were shown in Table 2. As shown in Table 2, there were more women in the unilateral nausea group than in the unilateral non-nausea group (male/female ratio: 80/192 versus 6/41, 
Table 1 Incidence of PONV after unilateral or bilateral THA/TKA

\begin{tabular}{lcccc}
\hline \multirow{2}{*}{ Time point } & \multicolumn{2}{c}{ Unilateral $(\mathrm{N}=319)$} & \multicolumn{2}{c}{ Bilateral $(\mathrm{N}=177)$} \\
\cline { 2 - 5 } & Nausea $(\mathrm{n} / \%)$ & Vomiting $(\mathrm{n} / \%)$ & Nausea $(\mathrm{n} / \%)$ & Vomiting $(\mathrm{n} / \%)$ \\
\hline 0-6 hours & $47 / 14.73 \%$ & $20 / 6.27 \%$ & $40 / 22.60 \%{ }^{\#}$ & $26 / 14.69 \%{ }^{*}$ \\
6-24 hours & $46 / 14.42 \%$ & $24 / 7.52 \%$ & $31 / 17.51 \%^{\$}$ & $19 / 10.73 \%^{\$}$ \\
24-72 hours & $16 / 5.02 \%$ & $7 / 2.19 \%$ & $11 / 6.21 \%^{\$}$ & $7 / 3.95 \%^{\$}$ \\
P value & $<0.001^{*}$ & $0.011^{*}$ & $0.001^{*}$ & $0.007^{*}$ \\
\hline
\end{tabular}

${ }^{*} \mathrm{P}$ values are significant among different time points in nausea and vomiting groups. Compared with unilateral surgery, ${ }^{\#} \mathrm{P}=0.0273$,

${ }^{\&} \mathrm{P}=0.002,{ }^{\$} \mathrm{P}>0.05$. PONV, postoperative nausea and vomiting; THA, total hip arthroplasty; TKA, total knee arthroplasty.
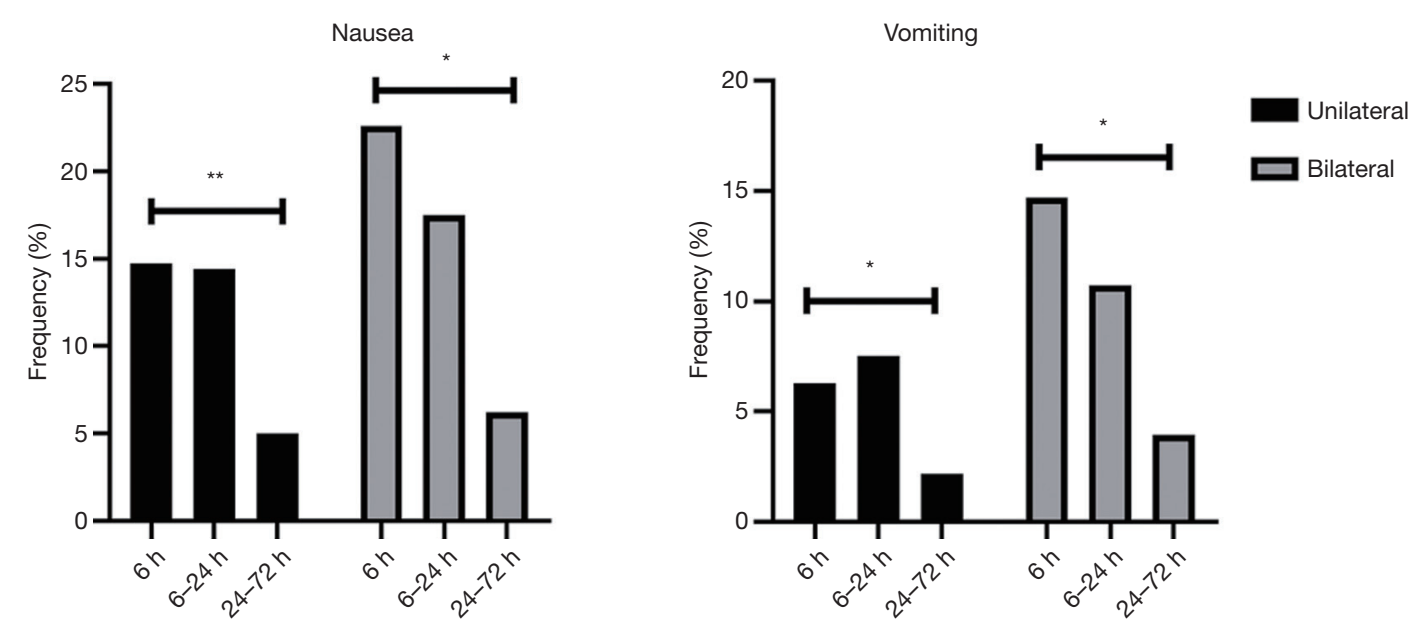

Figure 1 Incidence of PONV after THA/TKA. *, $\mathrm{P}<0.05$, significant among different time points in nausea and vomiting groups; **, $\mathrm{P}<0.001$, significant among different time points in nausea and vomiting groups. PONV, postoperative nausea and vomiting; THA, total hip arthroplasty; TKA, total knee arthroplasty.

$\mathrm{P}<0.05)$. There was a significant difference between nausea and non-nausea groups and between the vomiting and nonvomiting groups in motion sickness (all $\mathrm{P}<0.05)$. From the result of 6-24 hours, patients in the nausea group were younger than those in the non-nausea group and, similarly, patients in the vomiting group were younger than those in the nonvomiting group (all $\mathrm{P}<0.05$ ) after unilateral THA/ TKA. In patients who underwent bilateral THA/TKA, the vomiting group had lower preoperative $\mathrm{Hb}$ levels than the nonvomiting group (all $\mathrm{P}<0.05$ ). Patients with a migraine history were more likely to develop nausea $(\mathrm{P}<0.05)$. From the result of 24-72 hours, patients with a lower BMI were more likely to experience nausea after bilateral THA/TKA $(\mathrm{P}<0.05)$. Patients with PONV have a higher vomiting score (all $\mathrm{P}<0.05$ ). Further, patients using an analgesic pump tended to experience PONV at 24-72 hours after unilateral THA/TKA.
As shown in 0-6 and 6-24 hours, there were fewer patients with a smoking history in the unilateral nausea group than in the unilateral non-nausea group $(\mathrm{P}<0.05)$. Patients with higher VAS scores tend to have nausea after bilateral THA/TKA (all $\mathrm{P}<0.05$ ). The result of 6-24 and 24-72 hours showed that in patients who underwent bilateral THA/TKA, the vomiting group had lower preoperative $\mathrm{Hb}$ levels than the nonvomiting group (all $\mathrm{P}<0.05$ ).

\section{Binary logistic regression analysis}

Our earlier findings showed many patient- and surgicalrelated factors affecting PONV. Therefore, we analyze the risk factors of PONV after accounting for the effects of confounding factors at different time points after THA/ TKA. Age, obesity, and all other factors were adjusted when estimating the ORs with $95 \%$ confidence intervals of each 


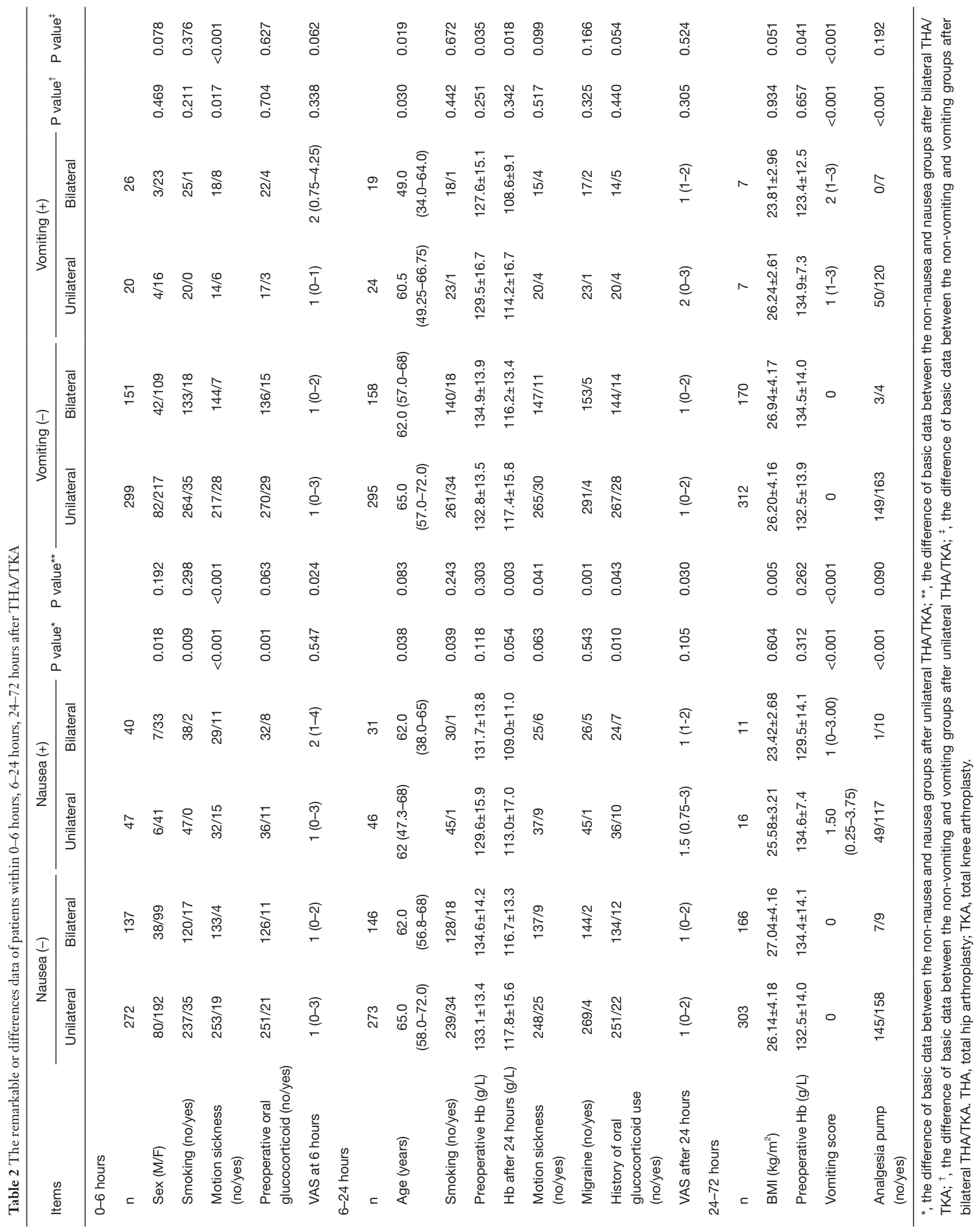


variable at 0-6 hours. Sex, obesity, and all other factors were adjusted when estimating the ORs with $95 \%$ confidence intervals of each variable at 6-24 hours. Sex, age, and all other factors were adjusted when estimating the ORs with 95\% confidence intervals of each variable at 24-72 hours. The results of binary logistic regression analysis are shown in Table 3. A stronger association of motion sickness and PONV was found at 0-6 hours after bilateral THA/TKA (nausea: $\mathrm{OR}=14.648,3.939-54.470$; vomiting: $\mathrm{OR}=8.405$, 2.482-28.466). Patients with a history of oral glucocorticoid use before unilateral surgery were more likely to experience nausea $(\mathrm{OR}=5.659,1.381-23.193)$. At 6-24 hours after bilateral THA/TKA, patients who had a history of migraines tended to experience nausea $(\mathrm{OR}=12.589,1.978-$ 80.105). Patients with higher VAS scores were more prone to experiencing nausea, with an OR of 1.283 at 6 hours after bilateral THA/TKA and 1.235 at $6-24$ hours after unilateral THA/TKA. Patients with higher vomiting scores were more likely to experience PONV at 24-72 hours, and those with lower BMI were more likely to have PONV (nausea: $\mathrm{OR}=0.767,0.616-0.954$; vomiting: $\mathrm{OR}=0.666$, 0.450-0.983) after bilateral THA/TKA.

\section{Discussion}

To our knowledge, this is the first study to analyze the risk factors of PONV divided into groups according to unilateral or bilateral surgery at different time points (0-6, 6-24, and 24-72 hours) after THA/TKA in a large sample. The risk factors affecting PONV vary at different time points after the operation. Our results showed that patient factors primarily affect the occurrence of postoperative PONV.

Concerning patient-related factors in the present study, the incidence of PONV is higher in women and nonsmokers, like that reported in previous studies $(15,16)$. It is believed that smoking may be related to the activation of dopamine receptors, one of the pathogenic mechanisms of nausea and vomiting. Moreover, there are fewer female smokers than male smokers, which may also explain why women are more likely to develop PONV than men. Perioperative anemia is common in orthopedic patients and is associated with adverse clinical outcome (17). Similarly, in our study, patients with low preoperative $\mathrm{Hb}$ levels were more likely to develop PONV from the result in 6-24 hours and 24-72 hours. It is better to minimize anemia and take measures for blood management when planning to perform THA/TKA to improve patient outcomes. The confounding factors were adjusted to analyze the potential risk factors using binary logistic regression analysis. A stronger association of motion sickness and PONV was found at 0-6 hours after bilateral THA/TKA. Patients with a history of motion sickness may have a low vomiting threshold and are prone to the re-occurrence of PONV. This high risk may be related to the abnormal sensitivity of vestibular function in such patients (18).

Earlier studies have found that BMI is positively associated with the occurrence of PONV, which may be the accumulation of fat-soluble anesthetics in fat tissue. After the anesthesia is stopped, the residual drug can still slowly enter the bloodstream, resulting in increased postoperative adverse effects. Interestingly, in our study, patients with lower BMI were more likely to have PONV at 24-72 hours after bilateral THA/TKA. Ukai et al. identified female sex, lower BMI, and use of opioids through an epidural catheter as factors likely to affect the occurrence of PONV (12). However, there are also results showing that increased BMI is not a risk factor of PONV (19).

Concerning surgical-related factors in our study, the results showed that after unilateral THA/TKA, the nausea frequency peaked at $14.73 \%$ at $0-6$ hours, and the vomiting frequency peaked at $7.52 \%$ at $6-24$ hours. However, after bilateral THA/TKA, the frequency of nausea and vomiting peaked at $22.60 \%$ and $14.69 \%$, respectively. The incidence of PONV after primary THA or TKA was higher after bilateral surgery than after unilateral surgery.

Therefore, when the data was analyzed, the patients were grouped according to unilateral and spontaneous bilateral surgery. When we compare the operation times, it is reasonable that there is no difference between the nonnausea and nausea groups or the nonvomiting and vomiting groups after unilateral or spontaneous bilateral THA/ TKA, because the entire surgical time is almost the same in unilateral or spontaneous bilateral THA/TKA.

PONV occurs mostly within 6 hours after surgery, suggesting that surgical stress or general anesthesia may increase the risk of PONV.

Concerning anesthesia-related factors in the present study, the preoperative anesthesia included propofol, sevoflurane, fentanyl, remifentanil, and rocuronium. Morphine is used for postoperative analgesia. However, there were no significant differences between the association of anesthesia time, morphine consumption, and PONV. These results may be related to the strict control of analgesics used in patients after THA/TKA at our hospital. Opioids can increase the sensitivity of the vestibular nervous system, inducing dizziness, and nausea in patients. Using 
Table 3 The risk factors of PONV factors at 0-6, 6-24, and 24-72 hours after THA/TKA using logistic regression analysis

\begin{tabular}{|c|c|c|c|c|c|c|}
\hline \multirow[b]{2}{*}{ Items } & \multicolumn{3}{|c|}{ Nausea } & \multicolumn{3}{|c|}{ Vomiting } \\
\hline & $\begin{array}{l}\text { Adjusted odds } \\
\text { ratio }\end{array}$ & $\begin{array}{c}95 \% \text { confidence } \\
\text { interval }\end{array}$ & $P$ value & $\begin{array}{l}\text { Adjusted odds } \\
\text { ratio }\end{array}$ & $\begin{array}{c}95 \% \text { confidence } \\
\text { interval }\end{array}$ & $P$ value \\
\hline \multicolumn{7}{|l|}{$0-6$ hours } \\
\hline \multicolumn{7}{|l|}{ Unilateral } \\
\hline Sex & 1.382 & $0.527-3.628$ & 0.511 & 0.718 & $0.219-2.346$ & 0.583 \\
\hline Motion sickness & 4.774 & $2.116-10.774$ & $<0.001$ & 3.798 & $1.287-11.205$ & 0.016 \\
\hline History of oral glucocorticoid use & 2.591 & $0.944-7.110$ & 0.362 & 1.631 & $0.361-7.367$ & 0.525 \\
\hline VAS at 6 hours & 1.084 & $0.911-1.291$ & 0.511 & 0.972 & $0.748-1.263$ & 0.832 \\
\hline \multicolumn{7}{|l|}{ Bilateral } \\
\hline Sex & 1.250 & $0.353-4.426$ & 0.729 & 2.291 & $0.411-12.768$ & 0.344 \\
\hline Motion sickness & 14.648 & $3.939-54.470$ & $<0.001$ & 8.405 & $2.482-28.466$ & 0.001 \\
\hline History of oral glucocorticoid use & 5.659 & $1.381-23.193$ & 0.016 & 2.376 & $0.457-12.363$ & 0.304 \\
\hline VAS at 6 hours & 1.283 & $1.077-1.528$ & 0.005 & 1.233 & $1.020-1.491$ & 0.030 \\
\hline \multicolumn{7}{|l|}{$6-24$ hours } \\
\hline \multicolumn{7}{|l|}{ Unilateral } \\
\hline Age & 0.977 & $0.952-1.002$ & 0.070 & 0.974 & $0.943-1.006$ & 0.110 \\
\hline Smoking & 0.160 & $0.018-1.392$ & 0.097 & 0.281 & $0.030-2.614$ & 0.264 \\
\hline Preoperative $\mathrm{Hb}$ & 0.998 & $0.967-1.031$ & 0.926 & 0.986 & $0.945-1.027$ & 0.493 \\
\hline $\mathrm{Hb}$ after 24 hours & 0.996 & $0.969-1.023$ & 0.757 & 1.003 & $0.966-1.042$ & 0.881 \\
\hline Motion sickness & 1.699 & $0.684-4.219$ & 0.253 & 1.267 & $0.373-4.307$ & 0.705 \\
\hline Migraine & 1.479 & $0.150-14.611$ & 0.738 & 3.033 & $0.301-30.535$ & 0.346 \\
\hline History of oral glucocorticoid use & 1.991 & $0.704-5.632$ & 0.194 & 1.098 & $0.265-4.556$ & 0.898 \\
\hline VAS after $24 \mathrm{~h}$ & 1.235 & $1.002-1.523$ & 0.048 & 1.174 & $0.902-1.527$ & 0.232 \\
\hline \multicolumn{7}{|l|}{ Bilateral } \\
\hline Age & 0.988 & $0.950-1.027$ & 0.544 & 0.959 & $0.917-1.003$ & 0.067 \\
\hline Smoking & 0.313 & $0.031-3.213$ & 0.328 & 0.597 & $0.052-6.799$ & 0.678 \\
\hline Preoperative $\mathrm{Hb}$ & 1.011 & $0.969-1.054$ & 0.624 & 0.967 & $0.922-1.015$ & 0.181 \\
\hline $\mathrm{Hb}$ after $24 \mathrm{~h}$ & 0.964 & $0.925-1.006$ & 0.089 & 0.990 & $0.941-1.041$ & 0.697 \\
\hline Motion sickness & 2.897 & $0.775-10.831$ & 0.114 & 2.558 & $0.583-11.233$ & 0.213 \\
\hline Migraine & 12.589 & $1.978-80.105$ & 0.007 & 3.015 & $0.452-20.087$ & 0.254 \\
\hline History of oral glucocorticoid use & 2.412 & $0.565-10.285$ & 0.234 & 1.148 & $0.231-5.692$ & 0.866 \\
\hline VAS after 24 hours & 1.261 & $0.949-1.676$ & 0.109 & 1.128 & $0.804-1.584$ & 0.485 \\
\hline \multicolumn{7}{|l|}{ 24-72 hours } \\
\hline \multicolumn{7}{|l|}{ Unilateral } \\
\hline BMI & 0.951 & $0.820-1.102$ & 0.506 & 0.992 & $0.806-1.220$ & 0.938 \\
\hline Analgesia pump & 1.024 & $0.973-1.077$ & 0.366 & 1.032 & $0.957-1.114$ & 0.413 \\
\hline Vomiting score & 1.571 & $1.187-2.078$ & 0.002 & 1.270 & $1.048-1.541$ & 0.015 \\
\hline \multicolumn{7}{|l|}{ Bilateral } \\
\hline BMI & 0.767 & $0.616-0.954$ & 0.017 & 0.666 & $0.450-0.983$ & 0.041 \\
\hline Analgesia pump & 0.977 & $0.930-1.026$ & 0.351 & 0.967 & $0.908-1.029$ & 0.290 \\
\hline Vomiting score & 1.718 & $1.080-2.733$ & 0.022 & 2.609 & $1.230-5.535$ & 0.012 \\
\hline
\end{tabular}


opioids in analgesia pumps has the potential to cause PONV. Our results showed that patients using an analgesia pump tended to experience nausea during 24-72 hours after unilateral THA/TKA. However, anesthesia drugs have been used in the prevention of acute postoperative pain and the occurrence of PONV (20). However, a study showed that the PONV frequency was estimated to be $37 \%$ in those treated with systemic morphine (21). Therefore, the effect of anesthesia drugs on PONV still needs further exploration.

We concluded from the analysis above, more active prevention for PONV is needed for women, non-smokers, and those with a history of motion sickness. At present, dexamethasone is the drug of choice for prevention and treatment (22). A recent meta-analysis including 60 randomized controlled trials with 6,696 subjects indicated a 4- to 5-mg dose regimen of systemic dexamethasone reduces the occurrence of PONV (23). However, in our study, there was no intravenous infusion of glucocorticoids, and patients with a history of oral glucocorticoid use who underwent unilateral THA/TKA were more likely to experience nausea. These results may be because patients with a history of oral glucocorticoid use may have some immune-related underlying conditions, and their lower physical ability makes them susceptible to developing PONV. Because PONV is a complex pathophysiological phenomenon involving the central and peripheral nervous systems, there is no single antiemetic agent that can block all receptors that cause nausea and vomiting. For high-risk patients, including those with motion sickness, multiple drugs should be used, including dexamethasone, 5-HT3 receptor antagonist, and droperidol $(24,25)$, in combination with nursing interventions including psychological nursing, preoperative gastrointestinal preparation, preoperative and postoperative diet, and activity guidance.

This study has several limitations. First, as this is a retrospective study, there may be information bias; for example, information on the history of motion sickness was self-reported by the patients. Second, some confounding factors were not controlled. Finally, this study only investigated the risk factors affecting PONV after THA/ TKA. Further studies that investigate the mechanisms that cause PONV are needed.

\section{Acknowledgments}

We thank all individuals, primary care doctors, and nurses who took part in the study. We would like to thank Editage (www.editage.cn) for English language editing.
Funding: Research grants funded this work from the National Natural Science Foundation of China (Grant No. 81630064 and 81871786; http://www.nsfc.gov.cn/).

\section{Footnote}

Reporting Checklist: The authors have completed the STROBE reporting checklist. Available at http://dx.doi. org/10.21037/atm-20-5486

Data Sharing Statement: Available at http://dx.doi. org/10.21037/atm-20-5486

Conflicts of Interest: All authors have completed the ICMJE uniform disclosure form (available at http://dx.doi. org/10.21037/atm-20-5486). The authors have no conflicts of interest to declare.

Ethical Statement: The authors are accountable for all aspects of the work in ensuring that questions related to the accuracy or integrity of any part of the work are appropriately investigated and resolved. The medical data in this study has been approved by the Institutional Review Board (IRB) of Peking Union Medical College Hospital (Protocol number: S-K1067). All procedures performed in this study involving human participants were in accordance with the Declaration of Helsinki (as revised in 2013). Because of the retrospective nature of the research, the requirement for informed consent was waived.

Open Access Statement: This is an Open Access article distributed in accordance with the Creative Commons Attribution-NonCommercial-NoDerivs 4.0 International License (CC BY-NC-ND 4.0), which permits the noncommercial replication and distribution of the article with the strict proviso that no changes or edits are made and the original work is properly cited (including links to both the formal publication through the relevant DOI and the license). See: https://creativecommons.org/licenses/by-nc-nd/4.0/.

\section{References}

1. Kovac AL. Updates in the Management of Postoperative Nausea and Vomiting. Adv Anesth 2018;36:81-97.

2. Ouanezar H, Jalaguier T, Franck F, et al. Mid-term outcomes of titanium modular neck femoral stems in revision total hip arthroplasty. Ann Transl Med 2019;7:92.

3. Gan TJ, Alexander R, Fennelly M, et al. Comparison of 
different methods of administering droperidol in patientcontrolled analgesia in the prevention of postoperative nausea and vomiting. Anesth Analg 1995;80:81-5.

4. Pollak RA, Gottlieb IJ, Hakakian F, et al. Efficacy and Safety of Intravenous Meloxicam in Patients With Moderate-to-Severe Pain Following Bunionectomy: A Randomized, Double-Blind, Placebo-controlled Trial. Clin J Pain 2018;34:918-26.

5. Birch S, Stilling M, Mechlenburg I, et al. Effectiveness of a physiotherapist delivered cognitive-behavioral patient education for patients who undergoes operation for total knee arthroplasty: a protocol of a randomized controlled trial. BMC Musculoskelet Disord 2017;18:116.

6. Hooper VD. SAMBA Consensus Guidelines for the Management of Postoperative Nausea and Vomiting: An Executive Summary for Perianesthesia Nurses. J Perianesth Nurs 2015;30:377-82.

7. Marques EM, Jones HE, Elvers KT, et al. Local anaesthetic infiltration for peri-operative pain control in total hip and knee replacement: systematic review and meta-analyses of short- and long-term effectiveness. BMC Musculoskelet Disord 2014;15:220.

8. Ienca R, Giardiello C, Scozzarro A, et al. Improving Nausea and Vomiting Post-Elipse Balloon: a Novel SingleDose Regimen of $300 \mathrm{mg}$ Netupitant/0.5 mg Palonosetron Hydrochloride. Obes Surg 2019;29:2952-6.

9. Sansonnens J, Taffe P, Burnand B. Higher occurrence of nausea and vomiting after total hip arthroplasty using general versus spinal anesthesia: an observational study. BMC Anesthesiol 2016;16:44.

10. Yang Q, Zhang Z, Xin W, et al. Preoperative intravenous glucocorticoids can decrease acute pain and postoperative nausea and vomiting after total hip arthroplasty: A PRISMA-compliant meta-analysis. Medicine (Baltimore) 2017;96:e8804.

11. Kim JK, Ro DH, Lee HJ, et al. Efficacy of Systemic Steroid Use Given One Day After Total Knee Arthroplasty for Pain and Nausea: A Randomized Controlled Study. J Arthroplasty 2020;35:69-75.

12. Ukai T, Ebihara G, Watanabe M. Opioid administration via epidural catheter is a risk factor for postoperative nausea and vomiting in total hip arthroplasty: A retrospective study. J Orthop Sci 2018;23:973-6.

13. Rodriguez-Merchan EC. Simultaneous bilateral total knee arthroplasty in hemophilia: is it recommended? Expert Rev Hematol 2017;10:847-51.

14. Gunaratne R, Pratt DN, Banda J, et al. Patient Dissatisfaction Following Total Knee Arthroplasty: A
Systematic Review of the Literature. J Arthroplasty 2017;32:3854-60.

15. Kovac AL. Updates in the Management of Postoperative Nausea and Vomiting. Adv Anesth 2018;36:81-97.

16. Chimbira W, Sweeney BP. The effect of smoking on postoperative nausea and vomiting. Anaesthesia 2000;55:540-4.

17. Maezawa K, Nozawa M, Yuasa T, et al. Postoperative hemoglobin and recovery of hip muscle strength after total hip arthroplasty. J Orthop 2018;15:886-8.

18. Cao X, White PF, Ma H. An update on the management of postoperative nausea and vomiting. J Anesth 2017;31:617-26.

19. Kim JH, Hong M, Kim YJ, et al. Effect of Body Mass Index on Postoperative Nausea and Vomiting: Propensity Analysis. J Clin Med 2020;9:1612.

20. Min BW, Kim Y, Cho HM, et al. Perioperative Pain Management in Total Hip Arthroplasty: Korean Hip Society Guidelines. Hip Pelvis 2016;28:15-23.

21. Colvin LA, Bull F, Hales TG. Perioperative opioid analgesiawhen is enough too much? A review of opioid-induced tolerance and hyperalgesia. Lancet 2019;393:1558-68.

22. Awad K, Ahmed H, Abushouk AI, et al. Dexamethasone combined with other antiemetics versus single antiemetics for prevention of postoperative nausea and vomiting after laparoscopic cholecystectomy: An updated systematic review and meta-analysis. Int J Surg 2016;36:152-63.

23. De Oliveira GS Jr, Castro-Alves LJ, Ahmad S, et al. Dexamethasone to prevent postoperative nausea and vomiting: an updated meta-analysis of randomized controlled trials. Anesth Analg 2013;116:58-74.

24. Odom-Forren J, Jalota L, Moser DK, et al. Incidence and predictors of postdischarge nausea and vomiting in a 7-day population. J Clin Anesth 2013;25:551-9.

25. DREAMS Trial Collaborators and West Midlands Research Collaborative. Dexamethasone versus standard treatment for postoperative nausea and vomiting in gastrointestinal surgery: randomised controlled trial (DREAMS Trial). BMJ 2017;357:j1455.

(English Language Editor: J. Chapnick)

Cite this article as: Wang Y, Yang Q, Lin J, Qian W, Jin J, Gao P, Zhang B, Feng B, Weng X. Risk factors of postoperative nausea and vomiting after total hip arthroplasty or total knee arthroplasty: a retrospective study. Ann Transl Med 2020;8(17):1088. doi: 10.21037/atm-20-5486 